case. The paravertebral abscess was associated to the disc involvement in 23 cases. Epiduritis was associated in 21 cases. Plain radiography, performed in in the majority of cases (63 cases, $94 \%$ ), demonstrated pathological pictures in $56(83.5 \%)$ patients. MRI, performed in $60(89.5 \%)$ patients, disease was in all patients. Pathogens were isolated in $43(64.1 \%)$ cases. Tuberculosis was the most common cause. The leading causative agents in non tuberculousspondylodiscitis were: Staphylococcus aureus (8 isolates, $11.9 \%$ ), brucella (7 isolates, $10.4 \%$ ), Escherichia coli (2 isolates, $2.9 \%$ ) and streptococcus B (1 isolates, 1.4\%). Two microorganisms combined (mycobacterium tuberculosis and a pyogenic) was found in one case. Medical treatment was adapted to the prescribed seed. Surgical treatment was performed in 6 patients. After therapy, $59(88 \%)$ patients had regression of symptoms, two patients had a permanent neurological impairment (paraplegia), one patient had recurrence of infection and one patient was dead.

Conclusions: Infectious spondylodiscitis has been diagnosed with increasing frequency. It should be taken into consideration in differential diagnosis in patients with significant back pain and laboratory evidence of an acute inflammatory process, especially metastatic spinal disease or inflammatory spondyloarthritis

References:

[1] Zimmerli W. Clinical practice.Vertebralosteomyelitis. N Engl J Med 2010 Mar; 362(11):1022-9.

[2] Cottle L, Riordan T. Infectious spondylodiscitis. J Infect 2008 June;56(6): 401-12.

Disclosure of Interest: None declared

DOI: 10.1136/annrheumdis-2017-eular.6916

\section{AB0922 CLINICAL MANIFESTATIONS AND OUTCOMES OF ACUTE SEPTIC ARTHRITIS IN SONGKLANAGARIND HOSPITAL: A 10-YEAR RETROSPECTIVE STUDY}

T. Yaowmaneerat, D. Aiewruengsurat. Internal Medicine, Prinice of Songkhla University, Hadyai, Songkhla, Thailand

Background: Septic arthritis is a rheumatologic emergency. Its delayed diagnosis and treatment cause joint morbidity and mortality. ${ }^{1}$ Cases involving antimicrobialresistant bacteria have been reported. ${ }^{2,3}$

Objectives: To determine the clinical manifestations and outcomes of septic arthritis, find the factors associated with mortality, and discover the incidence of drug-resistant organisms in our institution

Methods: A retrospective study was performed. Septic arthritis was defined as the presence of acute inflammatory arthritis indicated by a positive synovial fluid or synovial tissue culture for bacteria. A total of 116 septic arthritis patients, who visited Songklanagarind Hospital from January 2005 to December 2014, were reviewed.

Results: The patient median age was 58 (IQR: 46, 72). Sixty-one patients (52\%) were female. The median onset of symptoms and symptoms until diagnosis were 5 (IQR: 2, 7) and 6 (IQR: 3, 10) days, respectively. Eighty-eight cases (76.7\%) had underlying diseases that might predispose to joint infection. Sixty-nine cases $(59.5 \%)$ had pre-existing joint disease. Joint pain was the most common presenting symptom, and $58 \%$ of the cases had fever. The most common presentation was monoarthritis $(87 \%)$, which was predominantly associated $(78 \%)$ with knee joint involvement. The median synovial fluid leukocyte counts were 64,460 cells $/ \mu \mathrm{L}$ (IQR: 30,300; 129,000). Blood cultures were positive in 53 patients (49.1\%). Synovial fluid cultures commonly had Streptococcus spp. growth $(41 \%)$. Seven cases $(7 \%)$ involved drug-resistant organisms. All of them either were diagnosed with septic arthritis during hospitalization or had a history of previous surgery. Twenty-five percent of the cases obtained the empirical antibiotic, ceftriaxone, and 86 patients $(80 \%)$ underwent arthrotomy drainage. The mortality rate was $12 \%$, and its associated factors were cancer, liver disease and advanced age. Conclusions: Streptococcus spp. is an emerging cause of septic arthritis in Southern Thai patients. Physicians should be aware of this in patients presenting with fever and acute monoarthritis, particularly those with comorbidities and underlying joint diseases. The proper empirical antibiotic of choice is ceftriaxone. References:

[1] Garcia-Arias M, Balsa A, Mola EM. Septic arthritis. Best practice \& research Clinical rheumatology. 2011;25(3):407-21.

[2] Frazee BW, Fee C, Lambert L. How common is MRSA in adult septic arthritis? Annals of emergency medicine. 2009;54(5):695-700.

[3] Chao CM, Lai CC, Hsueh PR. Bacteriology of septic arthritis at a regional hospital in Southern Taiwan. Journal of microbiology, immunology, and infection = Wei mian yu gan ran za zhi. 2013;46(3):241-2.

Disclosure of Interest: None declared

DOI: 10.1136/annrheumdis-2017-eular.1737

\section{Fibromyalgia}

\section{AB0923 MEETING THE FIBROMYALGIA CRITERIA HAS A NEGATIVE IMPACT ON TNF INHIBITORS EFFICACY IN PATIENTS WITH AXIAL SPONDYLOARTHRITIS}

A. Dantu $^{1,2}$, J. Michaud ${ }^{2}$, M. Gauthier Prieur ${ }^{1}$, F. Da Silva ${ }^{1}$, S. Pouplin ${ }^{2}$,
T. Lequerre ${ }^{2}$, O. Vittecoq ${ }^{2}$, M. Verdet ${ }^{1} .{ }^{1}$ Rheumatology, Elbeuf Louviers Val de Reuil Hospital, Saint Aubin les Elbeuf; ${ }^{2}$ Rheumatology, Rouen University Hospital, Rouen, France

Background: Fibromyalgia and spondyloarthritis can coexist and the overlap between the two diseases could have consequences on TNF inhibitors efficacy. Objectives: To evaluate TNF inhibitors efficacy in patients with axial spondyloarthritis fullfilling or not fibromyalgia criteria.

Methods: Prospective observational bicentric study on 25 patients who met ASAS 2009 axial spondyloarthritis criteria. Fibromyalgia was defined by ACR 2010 fibromyalgia criteria or by $a \geq 5 / 6$ score of the Fibromyalgia Rapid Screening Tool. Following items were recorded before and after a 6 months treatment with TNF inhibitors: Visual Analog Scale for pain and for patient global disease activity, values of ESR and CRP, number of tender joints, MASES score, number of Yunus tender points. All patients filled a self-questionnaire with BASDAI, BASFI, FiRST and ACR 2010 fibromyalgia scale items using SSS and WPI. Criterion of judgment: an ASAS partial remission state was compared in patients with or without fibromyalgia.

Results: Of the 25 patients enrolled, 15 (60\%) fulfilled ACR 2010 fibromyalgia criteria and $9(36 \%)$ had a $>5 / 6$ FiRST score. The proportion of patients fulfilling an ASAS partial remission state was significantly lower in patients with fibromyalgia according to the ACR 2010 criteria ( $20 \%$ vs $70 \%, p=0,034)$ or to the FiRST score $(0 \%$ vs $62,5 \%, p=0,0028)$. These patients had more severe disease activity and physical fonction than the patients without fibromyalgia. In this study, some factors were related with absence of ASAS partial remission: female gender, prior TNF inhibitor failure, ACR 2010 fibromyalgia criteria positivity, to have more than 11 Yunus points and to have $a \geq 5 / 6$ FiRST score.

Conclusions: Meeting the fibromyalgia criteria might have an impact on ASAS partial remission state and on efficacy of TNF inhibitors in patients with axial spondyloarthritis. The FiRST score was more specific to predict an absence of ASAS partial remission than the ACR 2010 fibromyalgia criteria. TNF inhibitors should be used with circumspection in case of FiRST score $\geq 5 / 6$ in patients with axial spondyloarthritis.

References:

[1] Perrot S., Bouhassira D., Fermanian J., the CEDR (2010) Development and validation of the Fibromyalgia Rapid Screening Tool (FiRST). Pain 150(2):250-256.

[2] Wolfe F., Clauw DJ., et al. (2011) Fibromyalgia criteria and severity scales for clinical and epidemiological studies: a modification of the ACR Preliminary Diagnostic Criteria for Fibromyalgia. J Rheumatol 38:1113-1122.

Disclosure of Interest: None declared

DOI: 10.1136/annrheumdis-2017-eular.4310

\section{AB0924 THE ROLE OF SELF LIMITING BEHAVIOUR, DEPRESSION AND SLEEP IN THE SEVERITY OF FATIGUE IN PATIENTS WITH FIBROMYALGIA}

M. Fernandes ${ }^{1}$, M.P. Guzzo ${ }^{2}$, C. Iannuccelli ${ }^{2}$, L. Mallia ${ }^{1}$, F. Lucidi $^{3}$, M. Di Franco ${ }^{2}$, C. Violani ${ }^{1} .{ }^{1}$ Department of Psychology; ${ }^{2}$ Department of Internal Medicine and Medical Specialties; ${ }^{3}$ Department of Developmental and Social Psychology, "Sapienza" University, Rome, Italy

Background: Fatigue and sleep disturbances are prominent symptoms in Fibromyalgia (FM) and significantly affect the level of the patients' impairment. Some studies reported a synergic interaction of depression and poor sleep quality associated with fatigue (e.g. [1]); while Marques et al. [2] showed a significant association of the fatigue severity with a limiting behaviour self-regulatory style of the patients, i.e. reducing daily activities and excessive resting.

Objectives: The purpose of this cross-sectional study was to analyse the predictors of severity of fatigue in Italian patients with FM.

Methods: Outpatients with a FM diagnosis who fulfilled both ACR/EULAR 1990 and 2010 criteria [3,4], after a medical visit at the Fibromyalgia center at Sapienza University Hospital "Umberto I", were invited to participate in a study on their cognitions and behaviours wearing for one week a wrist actigraph (AMI Motionlogger Watch). Actigraphic sleep parameters were averaged over six days. After 7 days the participants returned the actigraph and answered a structured interview conducted by a trained psychologist which included validated scales measuring depression (Brief Symptom Inventory [5]), perceived fatigue (Checklist of Individual Strength [6]), sleep habits (Sleep Disorder Questionnaire [7]) and behaviour regulation patterns (All-or-nothing and Limiting behaviour scales from Behavioural Responses to Illness Questionnaire [8]). In the previous month and during the study, pharmacological and non-pharmacological treatments were unchanged.

Results: Actigraphic monitoring and structured interview were completed by 39 female FM patients, with a mean age of 44.9 years $(S D=8.55)$ and an illness mean duration of 6.5 years $(\mathrm{SD}=5.72)$. The majority of the patients reported insomnia complaints, and $29(74.4 \%)$ met the DSM criteria for chronic insomnia. Fatigue severity resulted as the best subjective measure of fatigue, and was positively and significantly correlated with self-management through limiting behaviour, and with Total Time in Bed (TTB) measured through actigraphy. The correlation between TTB and Total Time Slept (TTS) and depression were not significant. Hierarchical regression considering TTB, Depression and Limiting behaviour, showed that all these variables give a significant independent contribution to the prediction 